\title{
Safety, efficacy, and maneuverability of a self-propelled capsule endoscope for observation of the human gastro- intestinal tract
}

두(1) $(9$

\author{
Authors \\ Ohtsuka $^{2}$, Naotake Ohtsuka², Kazuhide Higuchi ${ }^{1}$ \\ Institutions \\ 1 Second Department of Internal Medicine, Osaka Medical \\ College, Takatsuki, Osaka, Japan \\ 2 Mu Ltd., Seta, Shiga, Japan
}

Kazuhiro Ota ${ }^{1}$, Yuichi Kojima ${ }^{1}$, Kazuki Kakimoto ${ }^{1}$, Sadaharu Nouda ${ }^{1}$, Toshihisa Takeuchi ${ }^{1}$, Yasunori Shindo ${ }^{2}$, Yoshitake

submitted 31.12 .2020

accepted after revision $\quad 3.5 .2021$

Bibliography

Endosc Int Open 2021; 09: E1391-E1396

DOI 10.1055/a-1507-4540

ISSN 2364-3722

(c) 2021. The Author(s).

This is an open access article published by Thieme under the terms of the Creative Commons Attribution-NonDerivative-NonCommercial License, permitting copying and reproduction so long as the original work is given appropriate credit. Contents may not be used for commercial purposes, or adapted, remixed, transformed or built upon. (https://creativecommons.org/licenses/by-nc-nd/4.0/)

Georg Thieme Verlag KG, Rüdigerstraße 14,

70469 Stuttgart, Germany

Corresponding author

Kazuhiro Ota, MD, PhD, Second Department of Internal

Medicine, Osaka Medical College, 2-7, Daigaku-machi,

Takatsuki, Osaka, 569-8686, Japan

Fax: +8172-683-6859

clash_kaz@yahoo.co.jp

\section{ABSTRACT}

Background and study aims We developed a selfpropelled capsule endoscope that can be controlled from outside the body with real-time observation. To improve the device, we conducted a clinical trial of total gastrointestinal capsule endoscopy in healthy subjects to ascertain whether our first-generation, self-propelled capsule endoscope was safe and effective for observing the entire human gastrointestinal tract.

Patients and methods After adequate gastrointestinal pretreatment, five healthy subjects were instructed to swallow a self-propelling capsule endoscope and the safety of a complete gastrointestinal capsule endoscopy with this device was assessed. We also investigated basic problems associated with complete gastrointestinal capsule endoscopy. Results No adverse effects of the magnetic field were identified in any of the subjects. No mucosal damage was noted in any of the subjects with the use of our first-generation, self-propelling capsule endoscope. We found that it took longer than expected to observe the stomach; the view was compromised by the swallowed saliva. The pylorus was extremely difficult to navigate, and the endoscope's fin sometimes got caught in the folds of the small intestine and colon. Conclusions To resolve the problems associated with the existing self-propelling capsule endoscope, it may be necessary to not only improve the capsule endoscopes, but also to control the environment within the gastrointestinal tract with medications and other means. Our results could guide other researchers in developing capsule endoscopes controllable from outside the body, thus allowing real-time observation.

\section{Introduction}

Although wireless capsule endoscopy is physically and mentally less stressful for patients compared to conventional upper gastrointestinal endoscopy or colonoscopy, it cannot be controlled from outside the body. To overcome this problem, we have developed a first-generation, self-propelled capsule endoscope (SPCE) (Mu Ltd., Shiga, Japan) that functions by being attached to an existing capsule endoscope (PillCam series, Covidien,
Dublin, Ireland). It is designed to accomplish the following two objectives: (1) total gastrointestinal endoscopy using a capsule endoscope; and (2) retrograde colon capsule endoscopy. We have published several papers on our development of a firstgeneration SPCE [1-3]. First, we reported the controllability of the SPCE within the stomach of a live dog [1]. In 2012, we presented the first successful total gastroenteroscopy procedure using SPCE in a human at the conference of the American 
Society for Gastrointestinal Endoscopy [4]. In 2015, using porcine stomach models, we established that the ability to find lesions and to examine them in detail depended on the SPCE's viewing angle and the frame rate of the images, respectively [2]. These results were considered applicable to the development of a capsule endoscope (CE), including SPCE, that can be controlled from outside the body and used for real-time observation. We then needed to conduct a clinical study to address the usefulness and safety of our first-generation SPCE, the results of which could then be used to develop and to perfect the next-generation SPCE. In addition, we believe that it is important to identify problems faced by researchers in other institutions who are working on other types of CEs controllable from outside the body, with real-time observation [5-8].

We conducted a clinical trial of total gastrointestinal capsule endoscopy to assess the safety and gastrointestinal observability of our first-generation SPCE in the human gastrointestinal tract.

\section{Patients and methods}

\section{Ethics}

This study was conducted in accordance with the 1975 Declaration of Helsinki (1983). The study protocol was approved by the Osaka Medical College Clinical Research Review Board (CRB5180010) and registered with the University Hospital Medical Information Network Clinical Trial Registry (UMIN000027805) and the Japan Registry of Clinical Trials (jRCTs052180023).

\section{First-generation SPCE and drive system}

In this study, we used the previously reported New MiniMermaid System ( Fig.1a, \Fig.1b) [1-3]. The first-generation SPCE was created by connecting a dedicated fin made of silicon resin with a micromagnet to the PillCam COLON2 video capsule (Covidien, Dublin, Ireland). The length of the fin is $19 \mathrm{~mm}$. The length and width of the entire SPCE are $50 \mathrm{~mm}$ and $11 \mathrm{~mm}$, respectively ( $\triangleright$ Fig. 1C). The external magnetic field is necessary for vibration of the SPCE's fin and three-dimensional (3D) control. When the micromagnet is placed in an alternating magnetic field, it vibrates. This vibration is then transmitted to the fin and is converted to a propelling force in water. Therefore, it is necessary to fill the stomach and intestines with water to control the SPCE. $3 \mathrm{D}$ control of the SPCE can be achieved by adjusting the magnetic field. In our experiments, an examiner controlled the SPCE with a dedicated controller while making observations using a real-time monitoring system (RAPID Access; Covidien, Dublin, Ireland) ( Video 1, 0.00-0.14).

\section{Study design}

This prospective, single-arm study enrolled healthy subjects to assess the safety and gastrointestinal observability of our firstgeneration SPCE for examining the entire human gastrointestinal tract. The inclusion criteria were as follows: age between 20 and 65 years at the time of providing consent; provision of free informed consent on the basis of full understanding of the study protocol; and no history of medication use for 1 month

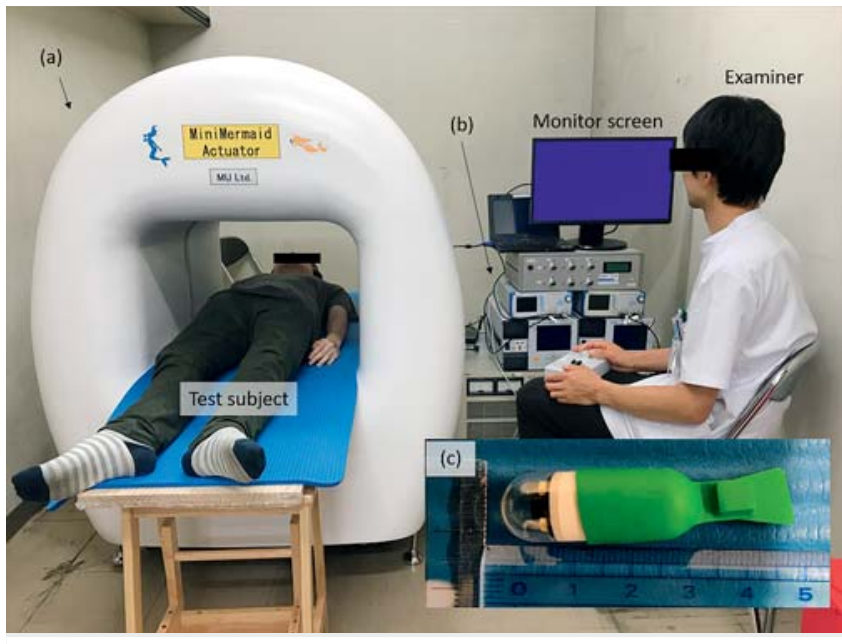

- Fig. 1a The patient was examined with the self-propelling capsule endoscope (SPCE) using the New MiniMermaid System. b Magnetic field generator. c Body of the SPCE.

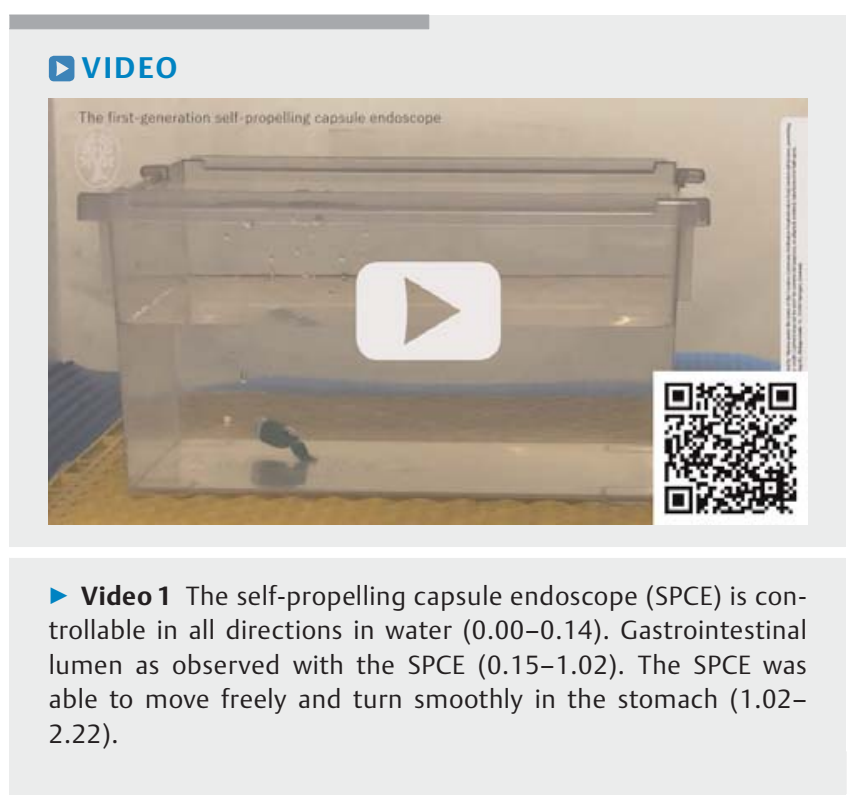

before enrollment. We excluded participants who had a history of gastrointestinal surgery other than appendectomy; used oral nonsteroidal anti-inflammatory drugs within 4 weeks before the study; had a history of gastrointestinal obstruction; did not consent to a surgery that would be required if the CE was retained in the body; had undergone implantation of a cardiac pacemaker or other electrical medical device; pregnant women; and those determined by the investigator, at his/her discretion, as being ineligible to participate in the study for any reason. Finally, five subjects were included. This study was conducted at the Osaka Medical College Hospital between October 2017 and November 2020. 
- Table 1 Records of transit time through each organ and problems with each subject.

\begin{tabular}{|c|c|c|c|c|c|c|c|}
\hline Number & $\begin{array}{l}\text { Age } \\
\text { (years) }\end{array}$ & $\begin{array}{l}\text { Discomfort during } \\
\text { swallowing }\end{array}$ & $\begin{array}{l}\text { Stomach } \\
\text { transit time }\end{array}$ & $\begin{array}{l}\text { Time for py- } \\
\text { lorus passage }\end{array}$ & $\begin{array}{l}\text { Small bowel } \\
\text { transit time }\end{array}$ & $\begin{array}{l}\text { Colon tran- } \\
\text { sit time }\end{array}$ & Problems \\
\hline 1 & 36 & $\begin{array}{l}+ \\
\text { (combined use of } \\
\text { swallowing jelly) }\end{array}$ & 20 & 42 & 241 & $\mathrm{n} / \mathrm{a}$ & $\begin{array}{l}\text { Visibility worsened due } \\
\text { to swallowing jelly } \\
\text { Transient retention at } \\
\text { hepatic flexure }\end{array}$ \\
\hline 2 & 30 & + & 12 & 72 & $\mathrm{n} / \mathrm{a}$ & $\mathrm{n} / \mathrm{a}$ & $\begin{array}{l}\text { Fin was broken in the } \\
\text { small intestine }\end{array}$ \\
\hline 3 & 34 & - & 24 & 17 & 184 & $\mathrm{n} / \mathrm{a}$ & $\begin{array}{l}\text { Transient retention at } \\
\text { ileocecal valve }\end{array}$ \\
\hline 4 & 32 & - & 19 & 138 & $\mathrm{n} / \mathrm{a}$ & $\mathrm{n} / \mathrm{a}$ & $\begin{array}{l}\text { Timeout in the small } \\
\text { intestine }\end{array}$ \\
\hline 5 & 40 & - & 16 & 75 & 194 & 93 & $\begin{array}{l}\text { Transient retention at } \\
\text { the rectum }\end{array}$ \\
\hline
\end{tabular}

\section{Protocol}

\section{Bowel preparation}

At 9 p.m. on the day before the examination, $24 \mathrm{mg}$ of sennoside was administered to each participant. On the day of the examination, $2 \mathrm{~L}$ of intestinal lavage solution (polyethylene glycol, Niflec, EA Pharma Co. Ltd. Tokyo, Japan) was consumed orally at 7 a.m. and the SPCE was swallowed at 10 a.m.

\section{Esophageal observation}

After the oral administration, the subject was placed in the supine position and the head was gradually raised to observe the esophagus. When the capsule reached the stomach, $500 \mathrm{~mL}$ of clear water (with a small amount of $2 \%$ Baros Antifoaming Oral Solution, dimethicone, Horii Pharmaceutical Ind., Ltd, Osaka, Japan) was administered.

\section{Observation of the stomach}

Beginning from the left lateral decubitus position, the SPCE was guided from the upper part of the stomach to the fundus and rotated several times using only a static magnetic field without movement. The SPCE was then moved from the upper part of the stomach to the fundus again. The patient was then placed in a supine position, and the same procedure was repeated as described above. In the right lateral decubitus position, the SPCE was rotated several times in the upper part of the stomach and observed. Then, the SPCE was guided toward the antrum in the supine and right lateral decubitus positions. The SPCE was then guided toward the duodenum in the supine and right lateral decubitus positions. Additionally, body position changes were made as deemed appropriate for the situation.

The small intestine was observed in the supine position. The fins continue to vibrate during small intestine observation for getting propulsion to shorten the observation time.

\section{Colon}

After reaching the cecum, $2 \mathrm{~L}$ of intestinal lavage solution was administered again and the cecum was observed in the supine position. Based on the endoscopic data received, the following parts were investigated: the ileocecal valve, hepatic flexure, splenic flexure, and the anus. The time to reach the rectum was approximately 6 hours after swallowing the CE.

\section{Endpoints}

The primary endpoint was the safety of the SPCE. The safety endpoints were determined as follows: vomiting or abdominal pain caused by intestinal obstruction due to $C E$, melena caused by mucosal damage due to the fin, and feeling ill secondary to the magnetic field.

The secondary endpoints were the transit time in each part of the gastrointestinal tract, the amount of bowel lavage required, and whether the images of the gastric cardia were obtained within 30 minutes. Because the cardia is the most difficult part of the stomach to observe [2], it was used as a marker of successful endoscopic observation of the entire stomach.

\section{Results}

All five subjects were healthy men, with a median age of 36 years (range: 30 to 40 years). The magnetic field used during the examination was deemed to be safe for each subject because there were no particular symptoms after the examination. The SPCE was ejected without any findings of damage to the gastrointestinal mucosa in each case, demonstrating that the first-generation SPCE was safe to use. $>$ Table 1 shows the transit time for SPCE within each part of the gastrointestinal tract of the subjects and the problems experienced in each case. 


\section{Discomfort on swallowing}

Although two of the five subjects complained of mild discomfort during swallowing, all subjects were able to swallow the SPCE without any problems. The first subject was asked to swallow the SPCE along with clear jelly to reduce discomfort during swallowing. However, the jelly was found to interfere with detailed observation of the stomach; hence, it was not used after the second patient.

\section{Observation in the stomach}

In three of five subjects, it was possible to identify the cardia or fundus in real time using SPCE. Due to the shape of the stomach, it was sometimes difficult to move the SPCE from the gastric body to the fundus. Observations from the gastric body to the antrum were easy and could be made in all cases. The degree of gastric cleansing by the bowel preparation protocol used was satisfactory. However, it was difficult to spend a long time observing the stomach because saliva flowing from the cardia reduced the visibility of the SPCE ( $\vee$ Video $1,0.15-0.34$ ).

\section{Time required to pass through the pylorus}

It took a substantial amount of time for the SPCE to pass through the pyloric sphincter. Under normal conditions, the inner diameter of the pyloric ring is smaller than the diameter of the SPCE. Therefore, the timing of manipulation of the SPCE through the pyloric ring had to be adjusted to correspond with the opening of the sphincter by peristalsis. This was very difficult to achieve because a median time of 72 minutes (range: 17 to 138 ) was required to navigate the pyloric ring alone $(\triangleright$ Video 1, 0.34-0.42).

\section{Transit within the small bowel}

In three subjects, SPCE passed through the entire small intestine with no delay. In the other two subjects, small bowel transit took longer than expected. This was a result of fin breakage due to the installation of a defective fin in one case; in another case, the fin of the SPCE was caught and stuck in the small bowel fold for some time. Mean small bowel transit time for SPCE in the three subjects in whom it passed through the entire small intestine flawlessly was 206.3 minutes (range: 184 to 241 ) ( Video 1, 0.42-0.51).

\section{Transit within the colon and the rectum}

In three of five subjects, the SPCE was able to reach the ileocecal valve, although the entire colon could be observed in only one subject. The colonic transit time of the SPCE in this subject was $93 \mathrm{~min}$. In one case, the fin was caught in the ileocecal valve and the SPCE could not pass through the colon due to a limited battery life. In another subject, the advancing part of the SPCE got stuck between the folds of the hepatic flexure. In each of the three cases in whom the SPCE reached the ileocecal valve, there was no turbidity in the bowel fluid and the colonic mucosa was clearly visualized ( $\vee$ Video $1,0.51-1.02$ ).

\section{Other problems}

All subjects had the urge to move their bowels during the examination due to the laxatives that were administered and this interrupted the real-time endoscopic observation several times.

\section{Supplementary experiment}

A similar pretreatment was performed on another subject. After swallowing the SPCE, its course in the stomach was observed using a nasal endoscope. When the stomach was filled with clear water, the SPCE was able to move freely and turn smoothly ( Video 1, 1.02-1.22). However, as the stomach contracted and the lumen narrowed due to peristalsis, the SPCE movement was more difficult ( $\vee$ Video 1, 1.22-1.52). Furthermore, it was difficult for the SPCE to pass through the gastric antrum as because there were multiple attempts to get through the pylorus ( $\triangleright$ Video $1,1.52-2.22$ ).

\section{Discussion}

This study demonstrates that the first-generation SPCE we developed is safe, as it was ejected without damaging the gastrointestinal mucosa in all of the subjects. Several reports about manipulation of CEs from outside the body have described the advantages and disadvantages of the respective devices. However, those results are not applicable to every device [5-8]. Here we discuss basic problems in operating CEs from outside the body based on results of this clinical study and suggest how future research and development should be conducted on that basis.

Naturally, capsule endoscopes do not have the suction, water infusion, or insufflation capabilities of conventional endoscopes and the accuracy of examination with them depends greatly on the environment within the gastrointestinal tract. Bowel preparation, therefore, is crucial to the success of the procedure.

Adequate expansion of the stomach is necessary to observe the stomach with the SPCE. The impression of direct endoscopic observation of the SPCE was that gastric peristalsis was stronger than expected. Gastric contractions not only inhibited movement of the capsule endoscope, but also propelled the water within the stomach into the duodenum. In our previous study, the extracted porcine stomach models that we used lacked peristalsis and expanded enough for observation by SPCE. Therefore, the entire stomach was observed in less than 10 minutes [2]. To obtain capsule endoscopic images that are comparable to those of conventional endoscopes, peristalsis must be sufficiently suppressed, and saliva must not be swallowed. In contrast, the clarity and amount of water that fills the stomach is also important. In this protocol, polyethylene glycol, which was used for cleansing, flushed out the gastric mucus; therefore, $500 \mathrm{~mL}$ of water was sufficient to ensure the expansion of the stomach and visibility of the SPCE. Ideally, we need to establish a method of observing the entire stomach in approximately 10 minutes. It was more difficult to observe the fundus and cardia than other parts on using the SPCE. Differences in the shape of the individual stomachs and other factors 
may have been responsible and undulations of the gastric wall may have adversely affected operation of the SPCE. This might be a basic problem for capsule endoscopes controllable from outside the body that allow real-time observation. Specific factors contributing to the difficulty of the SPCE in reaching the gastric fundus and cardia include inclination of the gastric wall, friction between the SPCE and gastric wall, and buoyancy of the SPCE. The most important aspect of the gastric observation is the examiner's ability to determine the location of SPCE in the stomach from the images, and development of instruments to assist with this aspect is currently underway at $\mathrm{Mu}$ Ltd. In addition, interruption of the gastric observation due to the need to defecate was a major problem; thus, other preparation methods need to be devised.

Second, the time taken by the SPCE to pass through the pylorus is an important problem. The current method takes substantial time to pass through the pylorus, and this issue needs to be resolved in order to make SPCE clinically applicable. Because the stomach is filled with water, it appears that the water is discharged from the stomach to the duodenum first and the capsule endoscope is then discharged into the duodenum. If the stomach is not filled with water, the SPCE may migrate more quickly to the duodenum; however, water is essential to stretch the stomach wall and avoid missing lesions between the folds. To allow SPCE to migrate to the duodenum even in the presence of water, it is necessary to consider amplifying the propulsive force of the SPCE and administrate suitable medications to relax the pyloric ring smooth muscle. The driving power of our SPCE was not enough to stimulate the pyloric sphincter to open. The physiologic relaxation of the pyloric sphincter occurs in a series of peristaltic movements from the stomach to the duodenum [9]. Although scopolamine butylbromide, an anticholinergic drug, has a relaxing effect on the pyloric ring [10], this medication is contraindicated in patients with glaucoma or arrhythmia and therefore has limited use. To power the SPCE to pass through the pylorus, one suggestion is to induce it with an additional magnetic field from outside the body. There is also a proposal to develop a smaller capsule endoscope; however, this is not currently practical.

Another issue was that the small intestine and colon were not completely observed. This was mainly caused by the fin getting stuck between the bends and folds of the intestinal tract. However, Mu Ltd. is currently developing the next generation of finless SPCE and we expect this issue to be resolved soon.

To solve the above-mentioned problems, it may be necessary to control the gastrointestinal environment not only by developing the capsule endoscopes themselves but also by using medications and other means. Our ultimate goal is not only to observe the gastrointestinal tract, but also perform mucosal sampling, topical drug application, and polyp excision. Observing the stomach lining with SPCE requires manipulation from outside the body, and observing inside the small intestine and colon can use intestinal peristalsis in the same way as with conventional capsule endoscopes. However, with the conventional method, the lesions of the small intestine and colon cannot be localized or treated. In the reports of both Rey et al. [5] and Cheng et al. [6], the CE was only magnetically controlled in the stomach and not for the observation of the small intestine and colon. It is possible that the observation was stopped in the small intestine because it would take too long for the capsule endoscope to pass through the pyloric ring. If those important issues are resolved, capsule endoscopy will gain more use in the future. Our goal is to overcome the problems associated with peristalsis in the entire gastrointestinal tract by self-propulsion. The observation of the entire gastrointestinal tract by the current SPCE requires a long duration and doctor's time as well. Improvements in equipment and training of doctors are essential to shorten the observation time, and integration with artificial intelligence is also under consideration.

There are several limitations to this study. The number of enrolled subjects was small. Of course, larger, more encompassing trials need to be performed. However, it was difficult to add more participants because of the COVID-19 pandemic. The subjects were young and different from the typical patients who undergo endoscopy of the digestive tract. This may have interfered with transit time. Fecal occult blood testing (FOBT) or a second capsule endoscopy is necessary to strictly check for microscopic mucosal injury and microscopic bleeding. We considered that FOBT to investigate the mild gastrointestinal bleeding or a second capsule endoscopy to check for lack of mucosal tearing was clinically unnecessary. The mild artificial mucosal injury caused by SPCE would resolve naturally and not be a clinical problem. Mucosal injury with massive bleeding or perforation would present as melena or a stomach ache. Finally, the next step is to evaluate the sensitivity of this kind of device under clinical conditions, which would be determined with a whole-digestive tract examination.

\section{Conclusions}

In conclusion, we have identified some basic problems associated with successful manipulation of capsule endoscopes from outside the body.

\section{Acknowledgements}

This work was supported by the Japan Society for the Promotion of Science KAKENHI Grant Number JP18K15829. The authors thank Shinya Nishida, Noriaki Sugawara, Kei Nakazawa, Yuta Yokoya, Hirota Miyazaki, Masanobu Fukumoto, Taro Iwatsubo, and Shimpei Kawaguchi, who cooperated in this study. They also thank Editage (www.editage.jp) for English language editing.

\section{Competing interests}

The authors declare that they have no conflict of interest.

\section{References}

[1] Morita E, Ohtsuka N, Shindo Y et al. In vivo trial of a driving system for a self-propelling capsule endoscope using a magnetic field (with video). Gastrointest Endosc 2010; 72: 836-840 
[2] Ota K, Nouda S, Takeuchi T et al. What kind of capsule endoscope is suitable for a controllable self-propelling capsule endoscope? Experimental study using a porcine stomach model for clinical application (with videos) PLoS One 2015; 10: e0139878

[3] Nouda S, Ota K, Higuchi K. Retrograde colon capsule endoscopy with the self-propelling capsule endoscope: The first human trial (with videos). Dig Endosc 2018; 30: 117-118

[4] Ohtsuka N, Umegaki E, Shindo Y et al. Observation of whole digestive tract of a human by a single passage of a self-propelling capsule endoscope. Gastrointest Endosc 2012; 75: AB126

[5] Rey JF, Ogata H, Hosoe N et al. Blinded nonrandomized comparative study of gastric examination with a magnetically guided capsule endoscope and standard videoendoscope. Gastrointest Endosc 2012; 75: $373-381$
[6] Cheng CS, Sun T], Zhang HD. Human gastric magnet-controlled capsule endoscopy conducted in a standing position: the phase 1 study. BMC Gastroenterol 2019; 19: 184

[7] Zou WB, Hou XH, Xin L et al. Magnetic-controlled capsule endoscopy vs. gastroscopy for gastric diseases: a two-center self-controlled comparative trial. Endoscopy 2015; 47: 525-528

[8] Qian YY, Zhu SG, Hou X et al. Preliminary study of magnetically controlled capsule gastroscopy for diagnosing superficial gastric neoplasia. Dig Liver Dis 2018; 50: 1041-1046

[9] Mochiki E, Kuwano H, Nakabayashi T et al. Pyloric relaxation regulated via intramural neural pathway of the antrum. Dig Dis Sci 2001; 46: 2307-2313

[10] Zhang L, Song J, Bai T et al. Effects of Buscopan on human gastrointestinal smooth muscle activity in an ex vivo model: Are there any differences for various sections? Eur J Pharmacol 2016; 780: 180-187 